\title{
Always think of the right ventricle, even in "low-risk" pulmonary embolism
}

\author{
Mareike Lankeit ${ }^{1,2}$
}

Affiliations: ${ }^{1}$ Dept of Internal Medicine and Cardiology, Campus Virchow Klinikum (CVK), Charité - University Medicine Berlin, Berlin, Germany. ${ }^{2}$ Center for Thrombosis and Hemostasis (CTH), University Medical Center of the Johannes Gutenberg-University Mainz, Mainz, Germany.

Correspondence: Mareike Lankeit, Department of Internal Medicine and Cardiology, Campus Virchow Klinikum (CVK), Charité - University Medicine Berlin, Augustenburger Platz 1, 13353 Berlin, Germany.

E-mail: mareike.lankeit@charite.de

\section{@ERSpublications}

RV dilation on MDCT is of prognostic relevance in apparently healthy and stable patients with pulmonary embolism http://ow.ly/pqFV30gQahd

Cite this article as: Lankeit M. Always think of the right ventricle, even in "low-risk" pulmonary embolism. Eur Respir J 2017; 50: 1702386 [https://doi.org/10.1183/13993003.02386-2017].

Patients with pulmonary embolism (PE) represent a prognostically heterogeneous patient population. Therefore, the current European Society of Cardiology (ESC) guidelines recommend risk stratification to guide risk-adjusted management [1]. Since no individual clinical, imaging or laboratory finding has been shown to predict risk of an adverse in-hospital outcome with adequate sensitivity and specificity to justify treatment decisions numerous studies have investigated combination models for an optimised risk classification [2,3]. Despite considerable progress made in the identification of intermediate-high-risk patients who might benefit from reperfusion therapy, there is an ongoing discussion whether patients classified as low-risk based on clinical parameters (such as the simplified pulmonary embolism severity index (sPESI) or the Hestia criteria) should undergo imaging or laboratory testing for right ventricular (RV) function. The ESC guidelines do not provide a clear recommendation, but suggest that "patients with a sPESI of 0 points and elevated cardiac biomarkers or signs of RV dysfunction on imaging test should be classified into the intermediate-low-risk category" and thus are not candidates for home treatment [1]. There are strong arguments for and against routine imaging or laboratory testing for RV function in low-risk patients: opponents argue that routine testing has not been shown to have therapeutic implications, is time- and cost-intensive, and does not provide additive prognostic information since the risk of adverse events is low per se in patients identified by the sPESI or the Hestia criteria. The study by CÔTé et al. [4] published in this issue of the European Respiratory Journal appears to support, at least in part, this notion. This pooled analysis of three prospective cohorts, namely the Prognostic Factors for Pulmonary Embolism (PREP) study, the PROgnosTic valuE of Computed Tomography scan in haemodynamically stable patients with acute symptomatic pulmonary embolism (PROTECT) study and a French prospective single-centre registry, confirms that the risk of PE-related adverse events and all-cause mortality during the first 30 days was low $(0.77 \%$ and $0.39 \%$, respectively) in 779 PE patients without relevant comorbidities (no cancer, cardiopulmonary disease and age $\leqslant 80$ years) and stable vital signs on admission (normal systolic blood pressure, heart rate and oxygen saturation) [4]. In this context, it should

Received: Nov 192017 | Accepted: Nov 202017

Support statement: This study was supported by the German Federal Ministry of Education and Research (BMBF 01EO1503). The author is responsible for the contents of this publication. Funding information for this article has been deposited with the Crossref Funder Registry.

Conflict of interest: Disclosures can be found alongside this article at erj.ersjournals.com 
be remembered that the 30 -day mortality rate was $1.0 \%$ in 305 "low-risk" patients of the sPESI derivation cohort and $1.1 \%$ in 2569 patients of the SPESI validation cohort [5].

Notwithstanding these findings, proponents argue that routine imaging or laboratory testing for RV dysfunction, which is considered the critical determinant of outcome in acute PE, should also be performed in patients without relevant comorbidities and (apparently) stable haemodynamic status on admission. Concerns against omitting RV testing are based on case reports demonstrating RV dysfunction in SPESI negative patients [6] and the premature discontinuation of a multicentre randomised trial by OTERO et al. [7] after the inclusion of 132 low-risk patients (identified by the Ursandi score) because of a $2.8 \%$ mortality rate in the early discharge group; of those, one young patient who had no transthoracic echocardiography died 5 days after the diagnosis of $\mathrm{PE}$ and autopsy revealed a large right heart thrombus. The VESTA study aimed to address this topic and randomised 550 PE patients without any of the Hestia criteria to direct discharge or additional N-terminal pro-brain natriuretic peptide (NT-proBNP) testing (and discharge if NT-proBNP was $\leqslant 500 \mathrm{ng} \cdot \mathrm{L}^{-1}$ or hospital admission if NT-proBNP was $>500 \mathrm{ng} \cdot \mathrm{L}^{-1}$ ). Although outpatient treatment of PE patients selected by the Hestia criteria alone was associated with a low rate of adverse events $(1.1 \%$ in the direct discharge group), the trial was unable to draw definite conclusions about the incremental value of NT-proBNP testing due to the low number of patients with elevated NT-proBNP levels [8].

The study by Côté et al. [4] is not only the largest cohort published to date focusing on low-risk patients (defined as an sPESI of 0 points), but is also the first investigating the impact of RV dysfunction assessed by multidetector computed tomography (MDCT) in this subgroup of patients. Some findings deserve special attention. Although the prevalence of RV dysfunction (defined as a right to left ventricular (RV/ $\mathrm{LV}$ ) diameter ratio $\geqslant 0.9$ ) was lower than in previous studies (for example, 66\% in unselected [9] and 63\% in normotensive [10] PE patients), a prevalence of 54\% appears considerably high in an apparently healthy and haemodynamically stable cohort of PE patients. Furthermore, although only three patients died during the first 30 days, all deaths were due to PE. Finally, although the mortality rate was not different in patients with an RV/LV diameter ratio $\geqslant 0.9$ compared to those with a RV/LV diameter ratio $<0.9(0.71 \%$ versus $0 \%, \mathrm{p}=0.254$ ), presumably due to the low number of events, all patients who died had a $\mathrm{RV} / \mathrm{LV}$ diameter ratio $>1.2$. By providing different $\mathrm{RV} / \mathrm{LV}$ diameter ratio thresholds, the authors were able to demonstrate an increase in specificity with increasing RV/LV diameter ratios and a significant difference in the rate of adverse events comparing patients with a RV/LV diameter ratio $\geqslant 1.1$ versus those with a $\mathrm{RV} / \mathrm{LV}$ diameter ratio <1.1 (details are provided in the supplementary material of the study by CôTÉ et al. [4]). These findings are in accordance with previous reports: a continuous increase of the RV/LV diameter ratio was identified as independent predictor of 30-day PE-related mortality in 785 unselected PE patients [11] and of 30-day complications in 190 normotensive PE patients [12].

So, how should prognostically relevant RV dysfunction on MDCT be defined? There is agreement that an increased RV/LV diameter ratio is associated with prognosis, both in unselected and in haemodynamic stable PE patients [13-15]. The most recent meta-analysis, including 49 studies with 13162 patients, reported a 2.5 -fold (95\% CI: 1.8-3.5) increased risk for all-cause mortality and a 5.0-fold (95\% CI: 2.7-9.2) increased risk for PE-related mortality in patients with increased RV/LV diameter ratios [15]. However, some studies were not able to demonstrate an association between RV/LV diameter ratios and prognosis, for example the PROTECT study with 848 normotensive patients who were also included in the present analysis by Côté et al. [4]. Differences in the prognostic performance might be related to the study design (studies with a retrospective design reported higher risk estimates [15]), progress and improvement of radiological techniques over the years, the methodology used for measuring RV and LV diameters (axial transverse versus reconstructed four-chamber views) and use of different thresholds to define an "abnormal" RV/LV diameter ratio. KANG et al. [16] observed, in 260 unselected PE patients, that a RV/LV diameter ratio measured in reconstructed four-chamber views was predictive of adverse outcomes while a $\mathrm{RV} / \mathrm{LV}$ diameter ratio measured in axial transverse sections was not. This finding was not confirmed by Lu et al. [17] who reported a similar prognostic performance for the two methods in 674 unselected patients. However, the choice of thresholds to define an "abnormal" RV/LV diameter ratio should be given more attention: considering the heterogeneity of PE patients with regard to comorbidities, symptoms and initial presentation, extent of RV dysfunction and prognosis the "one size fits all" concept for clinical, imaging and laboratory findings might no longer be sustainable. For example, the use of age-adjusted cut-off values for high-sensitivity troponin $\mathrm{T}\left(\geqslant 14 \mathrm{pg} \cdot \mathrm{mL}^{-1}\right.$ for patients aged $<75$ years and $\geqslant 45 \mathrm{pg} \cdot \mathrm{mL}^{-1}$ for patients aged $\geqslant 75$ years) was shown to improve risk assessment in 682 normotensive PE patients [18]. As demonstrated by Côté et al. [4], "mild" RV dilation (RV/LV diameter ratio $\geqslant 0.9$ ) due to the sudden increase of pulmonary artery pressure caused by the embolisation of thrombotic material to the pulmonary vasculature is a frequent finding with minor prognostic significance in patients without chronic cardiopulmonary disease, while a "more distinct" RV dilation (RV/LV diameter ratio $\geqslant 1.1$ ) indicates more 
severe PE, even if heart rate, systolic blood pressure and oxygen saturation are below the thresholds used for calculation of the sPESI. Finally, and importantly, MDCT provides more information on RV function than RV and LV diameters alone. While it is increasingly understood that thrombus load (for example assessed by the Qanadli, Mastora or modified Miller score) has only marginal if any prognostic implication [19] and the most recent studies were not able to demonstrate an association of central versus peripheral thrombus location with prognosis [20,21], evidence is accumulating that volumetric analysis of the heart chambers $[16,22,23]$ and measurement of contrast reflux to the inferior vena cava $[15,24,25]$ provide valuable prognostic information in patients with PE. For example, a left atrial volume $\leqslant 62 \mathrm{~mL}$ and right atrial/left atrial volume ratio $>1.2$ were associated with 30 -day mortality (adjusted hazard ratio 2.4, 95\% CI 1.5-3.9; $\mathrm{p}<0.001$ and adjusted hazard ratio 2.1, 95\% CI 1.3-3.4; $\mathrm{p}=0.002$, respectively) in 636 unselected patients with PE [23]. Furthermore, Kumamaru et al. [26] demonstrated, based on an analysis of 1698 MDCT scans from PE patients, that the combination of various information obtained from MDCT in a "CT-based prediction model" containing seven differently weighted variables allows reliable prediction of 30 -day mortality ( $0.0 \%$ in the lowest to $40.9 \%$ in the highest of four risk categories).

The following conclusion can be drawn based on the findings from Côté et al. [4]. First, since MDCT is the diagnostic modality of choice for confirming or excluding PE and is performed in the vast majority of patients, clinicians should seize the opportunity and use the information on the RV that is already available, even in "low-risk" PE patients. Secondly, future studies should focus on the definition of RV/LV diameter ratio thresholds for different subgroups of patients and the investigation and combination of further indicators of RV dysfunction to allow for a more differentiated risk assessment using MDCT.

\section{References}

1 Konstantinides SV, Torbicki A, Agnelli G, et al. 2014 ESC guidelines on the diagnosis and management of acute pulmonary embolism. Eur Heart J 2014; 35: 3033-3069.

2 Konstantinides SV, Barco S, Lankeit M, et al. Management of pulmonary embolism: an update. J Am Coll Cardiol 2016; 67: 976-990.

3 Meyer G, Planquette B, Sanchez O. Pulmonary embolism: whom to discharge and whom to thrombolyze? J Thromb Haemost 2015; 13: Suppl. 1, S252-S258.

4 Côté B, Jiménez D, Planquette B, et al. Prognostic value of right ventricular dilatation in patients with low-risk pulmonary embolism. Eur Respir J 2017; 50: 1701611.

5 Jiménez D, Aujesky D, Moores L, et al. Simplification of the pulmonary embolism severity index for prognostication in patients with acute symptomatic pulmonary embolism. Arch Intern Med 2010; 170: 1383-1389.

6 Hellenkamp K, Kaeberich A, Schwung J, et al. Risk stratification of normotensive pulmonary embolism based on the sPESI - does it work for all patients? Int J Cardiol 2015; 197: 162-163.

7 Otero R, Uresandi F, Jiménez D, et al. Home treatment in pulmonary embolism. Thromb Res 2010; 126: e1-e5.

8 den Exter PL, Zondag W, Klok FA, et al. Efficacy and safety of outpatient treatment based on the Hestia clinical decision rule with or without $\mathrm{N}$-terminal pro-brain natriuretic peptide testing in patients with acute pulmonary embolism. A randomized clinical trial. Am J Respir Crit Care Med 2016; 194: 998-1006.

9 Becattini C, Agnelli G, Vedovati MC, et al. Multidetector computed tomography for acute pulmonary embolism: diagnosis and risk stratification in a single test. Eur Heart J 2011; 32: 1657-1663.

10 Jiménez D, Lobo JL, Monreal M, et al. Prognostic significance of multidetector CT in normotensive patients with pulmonary embolism: results of the protect study. Thorax 2014; 69: 109-115.

11 George E, Kumamaru KK, Ghosh N, et al. Computed tomography and echocardiography in patients with acute pulmonary embolism: part 2: prognostic value. J Thorac Imaging 2014; 29: W7-W12.

12 Etesamifard N, Shirani S, Jenab Y, et al. Role of clinical and pulmonary computed tomography angiographic parameters in the prediction of short- and long-term mortality in patients with pulmonary embolism. Intern Emerg Med 2016; 11: 405-413.

13 Becattini C, Agnelli G, Germini F, et al. Computed tomography to assess risk of death in acute pulmonary embolism: a meta-analysis. Eur Respir J 2014; 43: 1678-1690.

14 Trujillo-Santos J, den Exter PL, Gómez V, et al. Computed tomography-assessed right ventricular dysfunction and risk stratification of patients with acute non-massive pulmonary embolism: systematic review and meta-analysis. $J$ Thromb Haemost 2013; 11: 1823-1832.

15 Meinel FG, Nance JW Jr, Schoepf UJ, et al. Predictive value of computed tomography in acute pulmonary embolism: systematic review and meta-analysis. Am J Med 2015; 128: 747-759.

16 Kang DK, Thilo C, Schoepf UJ, et al. CT signs of right ventricular dysfunction: prognostic role in acute pulmonary embolism. JACC Cardiovasc Imaging 2011; 4: 841-849.

17 Lu MT, Demehri S, Cai T, et al. Axial and reformatted four-chamber right ventricle-to-left ventricle diameter ratios on pulmonary CT angiography as predictors of death after acute pulmonary embolism. AJR Am J Roentgenol 2012; 198: 1353-1360.

18 Kaeberich A, Seeber V, Jiménez D, et al. Age-adjusted high-sensitivity troponin T cut-off value for risk stratification of pulmonary embolism. Eur Respir J 2015; 45: 1323-1331.

19 Vedovati MC, Germini F, Agnelli G, et al. Prognostic role of embolic burden assessed at computed tomography angiography in patients with acute pulmonary embolism: systematic review and meta-analysis. J Thromb Haemost 2013; 11: 2092-2102.

20 Senturk A, Ozsu S, Duru S, et al. Prognostic importance of central thrombus in hemodynamically stable patients with pulmonary embolism. Cardiol J 2017; 24: 508-514.

21 Jain CC, Chang Y, Kabrhel C, et al. Impact of pulmonary arterial clot location on pulmonary embolism treatment and outcomes (90 days). Am J Cardiol 2017; 119: 802-807. 
22 Aviram G, Sirota-Cohen C, Steinvil A, et al. Automated volumetric analysis of four cardiac chambers in pulmonary embolism: a novel technology for fast risk stratification. Thromb Haemost 2012; 108: 384-393.

23 Aviram G, Soikher E, Bendet A, et al. Prediction of mortality in pulmonary embolism based on left atrial volume measured on CT pulmonary angiography. Chest 2016; 149: 667-675.

24 Bach AG, Nansalmaa B, Kranz J, et al. CT pulmonary angiography findings that predict 30-day mortality in patients with acute pulmonary embolism. Eur J Radiol 2015; 84: 332-337.

25 Aviram G, Cohen D, Steinvil A, et al. Significance of reflux of contrast medium into the inferior vena cava on computerized tomographic pulmonary angiogram. Am J Cardiol 2012; 109: 432-437.

26 Kumamaru KK, Saboo SS, Aghayev A, et al. CT pulmonary angiography-based scoring system to predict the prognosis of acute pulmonary embolism. J Cardiovasc Comput Tomogr 2016; 10: 473-479. 